\title{
Higher dimensions and higher orders in EFT for gravitational waves
}

\author{
Ofek Birnholtz \\ Racah Institute of Physics, Hebrew University, Jerusalem, 91904, Israel \\ Albert-Einstein-Institut, Max-Planck-Institut für Gravitationsphysik, D-30167 Hannover, \\ Germany \\ E-mail: ofek.birnholtz@mail.huji.ac.il
}

\begin{abstract}
Techniques of effective field theory assist in finding previously unknown terms in the radiation-reaction effective action and self-force. We employ such methods to the analysis of gravitational waves emitted by and reacting on a binary system, in analogy to simpler systems. We present results for general dimensions to leading and $+1 \mathrm{PN}$, and discuss non-linear interactions.
\end{abstract}

Keywords: Radiation-reaction; self force; effective field theory; gravitational waves; postnewtonian.

\section{Introduction}

This work describes methods developed during $\mathrm{PhD}$ research ${ }^{1}$ about the gravitational waves (GW) emitted by and affecting the evolution of a gravitational binary system, including both published results ${ }^{2-6}$ as well as ongoing efforts. These join in an analytic development of an effective field theory (EFT) for radiation-reaction $(\mathrm{RR})$ in general relativity (GR).

In a spacetime of general dimension $d$, the Einstein-Hilbert action which couples the gravitational field $g_{\mu \nu}$ with the matter stress-energy tensor $T^{\mu \nu}$ can be given as

$$
S=\frac{1}{16 \pi G_{d}} \int \sqrt{-g} R d^{d} x-\frac{1}{2} \int h_{\mu \nu} T^{\mu \nu} d^{d} x
$$

with $R$ the Ricci scalar, $h_{\mu \nu}=g_{\mu \nu}-\eta_{\mu \nu}$ the metric perturbation, the (linearized) source satisfying $\nabla_{\mu} T^{\mu \nu}=0$, and under the usual convention $c=1$, but keeping explicitly the gravitational constant in $d$ dimensions, marked $G_{d}$, chosen with the normalization of the gravitational action so that the Newtonian potential is always $\Phi_{G}=-G_{d} M / r^{\hat{d}}$ where $\hat{d}=d-3$. Other conventions follow Ref. 5 .

When trying to calculate the radiation from the system and the back-reaction of the radiation on the system, we encounter several issues. First, there are numerous degrees of freedom in the metric, and in fact their number change with the dimensionality. These fields are coupled to one another, yet they have different properties, even mixing physical fields with pure gauge degrees of freedom. In addition, there are different symmetries relevant to the different zones where these fields are to considered, namely the system zone and the radiation zone. The dynamics itself is part conservative and part dissipative, losing energy to the radiation. Finally, the solution is sensitive to new effects as well as to increasing the order of different expansion parameters. 


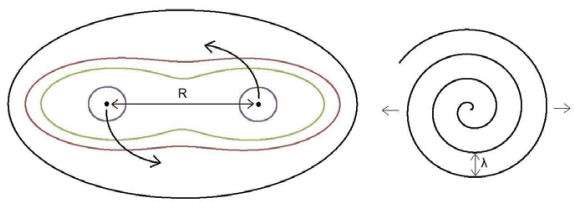

Fig. 1. Zone separation: system zone and radiation zone

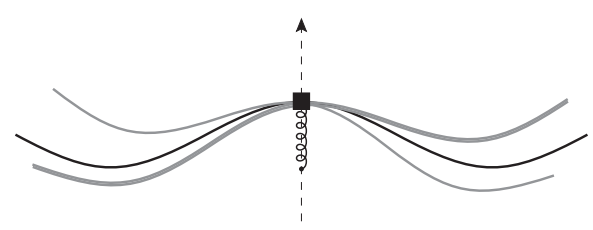

Fig. 2. Analogous system: a mass on a spring, attached to discrete set of $1 d$ strings

\section{Procedure Outline}

We separate the problem to two zones (Fig. 1): the internal System Zone, defined by the length scale $R$ of the orbital separation between bodies, and the Radiation Zone, with characteristics lengths given by the wavelengths $\lambda$. Under the regime of low velocities $v \ll c$ (post-Newtonian), $\lambda \sim \frac{R}{v} \gg R$. While the system zone is taken to be stationary, in the radiation zone time derivatives are of the order of the radial derivatives, but the symmetry is approximately (hyper-)spherical. Therefore we choose to describe the system zone using NRG fields ${ }^{8}$, and the radiation zone by (hyper-)spherical waves, and in particular by gauge-invariant (G.I.) combinations of spherical fields, which will serve as the Master fields $\mathfrak{h}_{S}, \mathfrak{h}_{V}, \mathfrak{h}_{T}$ (Scalar, Vector, Tensor). We match the Master fields to Master sources defined in the system zone, denoted $\mathcal{T}_{S}, \mathcal{T}_{V}, \mathcal{T}_{T}$ respectively. We then derive Master Actions and Master equations for the outgoing waves from the system.

This procedure reduces the $d$-dimensional system to a discrete set of $1+1$ dimensional (radial+time/frequency) radiation modes, coupled to corresponding point sources at the origin. This is analogous to a classical system of a mass at the origin, driven for example by a spring, and attached to a discrete set of outwards extending strings (Fig. 2); as the mass oscillates it radiates away waves along the strings to infinity ${ }^{3}$. We use Field Doubling to incorporate this radiation loss and its backreaction on the system in a Radiation-Reaction Effective Action - and derive various results from this effective action.

\section{Details for GR in General Dimension}

We start by decomposing the metric perturbation to Fourier basis in time, and to a basis of (hyper-)spherical harmonics in multi-index notation for the spatial coordinates ${ }^{5}$. We especially note the three distinct tensorial behaviours and their Youngtableux representations, Scalar $(\square \cdot \cdots \cdot)$, Vector $(\square \cdot 1 \cdot)$ and Tensor $(\square \cdot \Gamma){ }^{7}$.

$$
h_{\alpha \beta}=\sum_{L, \omega}\left(\begin{array}{ccc}
h_{t t} n_{L} & h_{t r} n_{L} & h_{t} \partial_{\Omega} n_{L}+h_{t V \aleph} n_{\aleph \Omega}^{L} \\
\cdots & h_{r r} n_{L} & h_{r} \partial_{\Omega} n_{L}+h_{r V \aleph} n_{\aleph \Omega}^{L} \\
\cdots & \cdots & h_{S} n_{\Omega \Omega^{\prime}}^{L}+\tilde{h}_{S} \tilde{n}_{\Omega \Omega^{\prime}}^{L}+h_{V \aleph} n_{\aleph \Omega \Omega^{\prime}}^{L}+h_{T \aleph \beth} n_{\aleph ב \Omega \Omega^{\prime}}^{L}
\end{array}\right) e^{-i \omega t},
$$

We find the combinations forming gauge-invariant Master Fields. We start with the tensor Master fields,

$$
\mathfrak{h}_{T \aleph ב}=r^{-(\ell+2)} h_{T \aleph ב} .
$$


Using 1 algebraic equation we also find the vector Master fields

$$
\mathfrak{h}_{V \aleph}=-\frac{\ell(\ell+\hat{d}+1)}{4 r^{\ell}}\left(\omega^{2}-\frac{\hat{c}_{s}}{r^{2}}\right)^{-1}\left(\frac{2 h_{t V \aleph}+i \omega h_{V \aleph}}{r^{2}}\right)^{\prime} .
$$

Next we use 3 algebraic equations to find the scalars $\Phi:=h_{t t}+2 i \omega h_{t}-\omega^{2} \tilde{h}_{S}$, canonically transformed to $\tilde{\Phi}$ with $F\left[\Phi, \Phi^{*}, \tilde{\Phi}, \tilde{\Phi}^{*}\right]=-\hat{c}_{s} r^{\frac{\hat{d}-1}{2}}\left(\tilde{\Phi}^{*} \Phi+\tilde{\Phi} \Phi^{*}\right)$, and finally the scalar Master field (more details in Ref. 5)

$$
\mathfrak{h}_{S}=-\frac{\hat{d}(\ell+\hat{d}+1)}{2 \ell} r^{-\left(\ell+\frac{\hat{d}+1}{2}\right)} \tilde{\Phi}
$$

The Master Action for these three Master Field families (for $\epsilon \in\{S, V, T\}$ ) is

$$
S^{\epsilon}=\frac{1}{2} \sum_{L, \omega} \int d r\left[\frac{N_{\ell, \hat{d}}}{G_{d} R_{\ell, \hat{d}}^{\epsilon}} r^{2 \ell+\hat{d}+1} \mathfrak{h}_{\epsilon}^{*} \mathfrak{L} \mathfrak{h}^{\epsilon}-\left(\mathfrak{h}_{\epsilon}^{*} \mathcal{T}^{\epsilon}+\text { c.c. }\right)\right],
$$

with

$$
\begin{aligned}
& N_{\ell, \hat{d}}=\frac{\Gamma(1+\hat{d} / 2)}{2^{\ell} \Gamma(1+\alpha)}=\frac{\hat{d} ! !}{(2 \ell+\hat{d}) ! !}, \quad \alpha=\ell+\frac{\hat{d}}{2}, \quad M_{\ell, \hat{d}}=\frac{\pi}{2^{2 \alpha+1} N_{\ell, \hat{d}} \Gamma^{2}(\alpha+1)} \\
& R_{\ell, \hat{d}}^{S}=\frac{\hat{d}(\ell+\hat{d}+1)(\ell+\hat{d})}{(\ell-1) \ell}, R_{\ell, \hat{d}}^{V}=\frac{(\hat{d}+1)(\ell+\hat{d}+1) \ell}{2(\ell-1)(\ell+\hat{d})}, R_{\ell, \hat{d}}^{T}=\frac{8(\hat{d}+1)}{\hat{d}^{2} c_{s}\left(c_{s}-\hat{d}\right)} .
\end{aligned}
$$

As the Actions are of the same form, so are the derived Master Wave Equations

$$
0=\frac{\delta S}{\delta \mathfrak{h}_{\epsilon}^{L \omega *}}=\frac{N_{\ell, \hat{d}}}{G_{d} R_{\ell, \hat{d}}^{\epsilon}} r^{2 \ell+\hat{d}+1}\left(\omega^{2}+\partial_{r}^{2}+\frac{2 \ell+\hat{d}+1}{r} \partial_{r}\right) \mathfrak{h}_{L \omega}^{\epsilon}-\mathcal{T}_{L \omega}^{\epsilon} .
$$

To treat the dissipation of radiation, we double the fields $\mathfrak{h} \rightarrow \hat{\mathfrak{h}}$ and the sources $Q \rightarrow \hat{Q}=\frac{\delta Q}{\delta x} \hat{x}^{9,10}$. The doubled sources reflect directed propagation (outwards), and may be interpreted as radiation "sinks". The doubled action is then given by

$$
\begin{aligned}
\hat{S}[\mathfrak{h}, \hat{\mathfrak{h}} ; Q, \hat{Q}] & =\int\left[\frac{\delta S}{\delta \mathfrak{h}} \hat{\mathfrak{h}}+\frac{\delta S}{\delta Q} \hat{Q}\right] \\
& =\int \hat{\mathfrak{h}}_{\epsilon}^{*}\left[\frac{N_{\ell, \hat{d}}}{G_{d} R_{\ell, \hat{d}}^{\epsilon}} r^{2 \ell+\hat{d}+1}\left(\omega^{2}+\partial_{r}^{2}+\frac{2 \ell+\hat{d}+1}{r} \partial_{r}\right) \mathfrak{h}^{\epsilon}-Q_{\epsilon}\right] .
\end{aligned}
$$

We use this action to read off the Feynman Rules. The homogeneous part defines a wave operator, whose inversion gives the directed Feynman propagator

$$
\int_{r^{\prime}}^{L}=G_{r e t}^{\epsilon}\left(r^{\prime}, r\right)=-i G \omega^{2 \ell+\hat{d}} M_{\ell, \hat{d}} R_{\ell, \hat{d}}^{\epsilon} \tilde{j}_{\alpha}\left(\omega r_{<}\right) \tilde{h}_{\alpha}^{+}\left(\omega r_{>}\right) \delta_{L L^{\prime}}
$$

in terms of the normalized Bessel functions

$$
\begin{aligned}
& \tilde{j}_{\alpha}:=\Gamma(\alpha+1) 2^{\alpha} \frac{j_{\alpha}(x)}{x^{\alpha}}=\sum_{p=0}^{\infty} \frac{(-)^{p}(2 \alpha) ! !}{(2 p) ! !(2 p+2 \alpha) ! !} x^{2 p}=1-\frac{x^{2}}{2(2 \alpha+2)}+\cdots, \\
& \tilde{h}_{\alpha}:=\Gamma(\alpha+1) 2^{\alpha} \frac{h_{\alpha}(x)}{x^{\alpha}} .
\end{aligned}
$$


The inhomogeneous part of the action gives the source and "sink" verteces, where the doubled vertical lines represent word-lines of the system zone components:

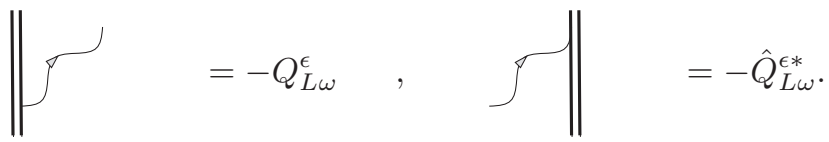

Together these make up the Radiation-Reaction Effective Action,

$$
\hat{S}=\| \approx=\sum(\hat{Q} G Q)
$$

which can then be variationally differentiated to produce the radiation-reaction on the system, namely the Self-Force $\mathbf{F}=\frac{\delta \hat{S}}{\delta \hat{\mathbf{x}}}$.

\section{Results}

The system-zone interactions are packed into the verteces of the radiation zone diagrams. Matching the terms that couple to the various $\mathfrak{h}_{L \omega}^{\epsilon}$ modes, we gather the source multipoles order-by-order in PN. For $n$ masses $m_{A}$, the first few are:

- Mass quadrupole $Q_{S}^{i j}=\sum_{A=1}^{n} m_{A}\left(x^{i} x^{j}-\frac{1}{D} \delta^{i j} x^{2}\right)_{A}$,

- Current quadrupole $\left.Q_{M}^{L_{2}}=Q_{M}^{i j}=2 \sum_{A=1}^{n}\left[m(\vec{r} \wedge \vec{J})^{(i} x^{j}\right)\right]_{A}$,

- Mass octupole

$$
Q_{S}^{L_{3}}=Q_{S}^{i j k}=\sum_{A=1}^{n} m_{A}\left[x^{i} x^{j} x^{k}-\frac{1}{D+2}\left(\delta^{i j} x^{k}+\delta^{i k} x^{j}+\delta^{j k} x^{i}\right) x^{2}\right]_{A} .
$$

We assemble the RR effective action from these verteces and the propagator,

$$
\begin{aligned}
\hat{S}_{\text {linear }}= & \|\|_{\ell}^{h_{S}} \| \\
=\int d t \sum_{\ell} \frac{G_{d}(-)^{\ell+\hat{d}}(\ell+\hat{d}+1)}{\hat{d} ! !(2 \ell+\hat{d}) ! !(\ell-1)} & \frac{\hat{d}(\ell+\hat{d})}{\ell} \hat{Q}_{(E)}^{L} \partial_{t}^{2 \ell+\hat{d}} Q_{L}^{(E)} \\
& \left.+\frac{(\hat{d}+1) \ell}{2(\ell+\hat{d})} \hat{Q}_{(M)}^{L} \partial_{t}^{2 \ell+\hat{d}} Q_{L}^{(M)}+\#(T)\right] .
\end{aligned}
$$

In particular, we record the leading order (LO) (compare Ref. 11\&12),

$$
S_{\text {eff }}^{L O}=(-)^{\frac{\hat{d}+1}{2}} G_{d} \int d t \frac{\hat{d}(\hat{d}+2)(\hat{d}+3)}{2 \hat{d} ! !(\hat{d}+4) ! !} \hat{Q}_{S}^{L_{2}} \partial_{t}^{d+1} Q_{S}^{L_{2}} \text {, }
$$


and the next-to-leading order (NLO),

$$
\begin{aligned}
S_{e f f}^{N L O}=(-)^{\frac{\hat{d}+1}{2}} G_{d} \int d t & {\left[\frac{\hat{d}(\hat{d}+2)(\hat{d}+3)}{2 \hat{d} ! !(\hat{d}+4) ! !}\left(\hat{Q}_{S}^{L_{2}} \partial_{t}^{d+1} \delta^{1} Q_{S}^{L_{2}}+\delta^{1} \hat{Q}_{S}^{L_{2}} \partial_{t}^{d+1} Q_{S}^{L_{2}}\right)\right.} \\
& \left.-\frac{\hat{d}(\hat{d}+4)(\hat{d}+3)}{6 \hat{d} ! !(\hat{d}+6) ! !} \hat{Q}_{S}^{L_{3}} \partial_{t}^{d+3} Q_{S}^{L_{3}}+\frac{2 \hat{d}(\hat{d}+3)}{3 \hat{d} ! !(\hat{d}+4) ! !} \hat{Q}_{M}^{L_{2}} \partial_{t}^{d+1} Q_{M}^{L_{2}}\right] .
\end{aligned}
$$

This NLO result for general dimension goes beyond any previous results; it may be compared to previous results in $4 \mathrm{~d}$ using the specialization

$$
\hat{S}_{L O+N L O}^{(d=4)}=G_{d} \int d t\left[-\frac{1}{5} \hat{Q}_{E}^{i j} \partial_{t}^{5} Q_{E}^{i j}-\frac{4}{45} \hat{Q}_{M}^{i j} \partial_{t}^{5} Q_{M}^{i j}+\frac{1}{189} \hat{Q}_{E}^{i j k} \partial_{t}^{7} Q_{E}^{i j k}\right] .
$$

\section{Higher Order Interactions}

\subsection{In System Zone: Quadrupole Moments (LO, NLO)}

The first non-linear effect is the correction to the Mass Quadrupole $\delta^{1} Q_{E}^{i j}$ from the gravitating potential energy, of order $\sim-\frac{G m_{A} m_{B}}{r^{\tilde{d}}}$ (which is $+1 \mathrm{PN}$ ), given by

$$
\begin{aligned}
\mid & =\delta^{1} Q_{S}^{L_{2}}=\sum_{A=1}^{n} m_{A}\left[\left(\frac{\hat{d}+2}{2 \hat{d}} v_{A}^{2}-\sum_{B \neq A} \frac{G_{d} m_{B}}{\left\|\vec{x}_{A}-\vec{x}_{B}\right\|^{\hat{d}}}\right) x_{A}^{L_{2}}\right. \\
& \left.-\frac{2(\hat{d}+1)}{\hat{d}(\hat{d}+2)} \partial_{t}\left(\vec{x}_{A} \cdot \vec{v}_{A} x_{A}^{L_{2}}\right)+\frac{\left(\hat{d}^{2}+6 \hat{d}+4\right)}{2 \hat{d}(\hat{d}+2)(\hat{d}+6)} \partial_{t}^{2}\left(r_{A}^{2} x_{A}^{L_{2}}\right)\right] .
\end{aligned}
$$

Higher orders of course include higher scalar and vector multipoles (Octupoles, hexadecapoles, etc.), as well as tensorial quadrupoles and higher. Similar (and higher) non-linear corrections also exist for those.

\subsection{Background interactions in Radiation Zone}

Having simplified the problem first by splitting between the system and radiation zones, then by defining the master fields in the radiation zone, we may incorporate new effects in a simple manner. While radiation modes may in fact interact with each-other, the leading interactions in the radiation zone are between individual radiation modes and the background curvature. The first of these is the scattering of a Scalar radiation mode off of the constant cosmological curvature of the background, represented by $\Lambda$, the Cosmological Constant. $\Lambda$ is expected to be very small, but need not be zero - and so these diagrams are suppressed by a factor $\Lambda$ with respect to the leading quadrupolar radiation. The leading Scalar quadrupole mode can also scatter off of the long-range (radiation-zone) curvature of the total mass of the system. To leading order, this is equivalent to taking the background to be static Schwarzschild rather than flat, with the mass $M$ the total 
mass of the source system, and calculating the leading correction to the radiationzone propagator on this background. As this interaction is in the radiation zone where $r \sim \lambda \sim \omega^{-1} \sim \frac{R}{v}$, the strength of gravitational potential is $\Phi_{G} \sim \frac{G_{d} M}{\lambda^{\tilde{d}}}$, and thus this interaction is suppressed w.r.t the leading quadrupole by a factor of order $\frac{G_{d} M}{R^{\hat{d}}}\left(\frac{R}{\lambda}\right)^{\hat{d}}$, i.e $+\left(1+\frac{\hat{d}}{2}\right) P N$ order. A similar interaction exists between the total mass curvature and the leading Vector quadrupole radiation mode, which enters at $+\left(2+\frac{\hat{d}}{2}\right) P N$ order. The contributions of these three interactions to the effective action are represented by the diagrams in Fig. 3. Not shown are $+\left(2+\frac{\hat{d}}{2}\right) P N$ order contributions from next-to-leading Scalar modes (octupole and corrections to the quadrupole, as described above).
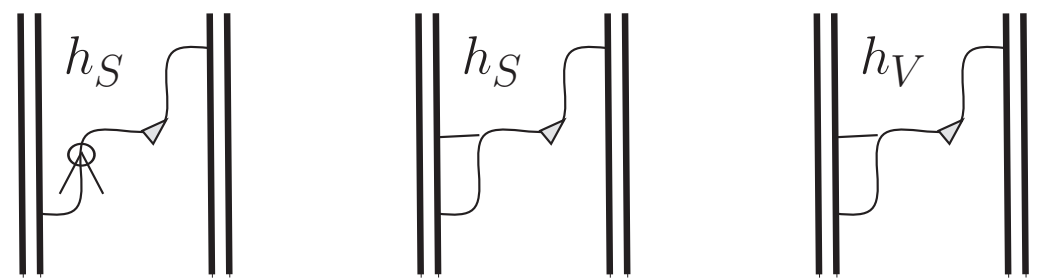

Fig. 3. Possible interactions of the radiation fields with the background: Scalar / Cosmological Constant $\Lambda$ (left), Scalar / Total Mass Curvature (center), Vector / Total Mass Curvature (right).

\subsection{Spin effects}

The discussion above has not yet included the effects of spin. While in $d=4$ the first spin-orbit couplings can enter at $+1.5 P N$ and spin-spin effects at $+2 P N$, as the dimension increases spin becomes more and more important. At $d=5$ spinorbit and spin-couplings can both enter at $+1 P N$, and in higher dimensions the spin-spin coupling enters at lower $\mathrm{PN}$ order than spin-orbit, which itself comes in below $+1 P N$, and these thus become the next-to-leading terms. The following table sums the PN orders of various spin terms, for the cases of co-rotation and of maximal spin.

\begin{tabular}{ccc}
\hline & co-rotation & maximal-spin \\
\hline Spin-Orbit & $1+2 / \hat{d}$ & $1 / 2+1 / \hat{d}$ \\
Spin-Spin & $4 / \hat{d}$ & $2 / \hat{d}$ \\
\hline
\end{tabular}

\section{Summary \& Future}

The method and formalism developed allows for a joint analytical Action formulation of radiation \& reaction. Such a formalism manifestly incorporates the symmetries of the problem and the relations and conservation laws between the system and the outgoing radiation. The action formalism, using Feynman diagrams, also economizes traditional computations. As the method reduces the dimension of spacetime 
2006

to a simpler $1+1 \mathrm{~d}$ problem, it is equally useful in any dimension. It has produced for the first time the Leading Order and Next-to-leading Order action, energy dissipation, and self-force for the GR 2-body problem in any dimension, as well as treatments of non-linear effects (separately for system and radiation zones). It is beginning to serve for analysis of some higher order effects in any dimension, and is a ready platform for other effects as well.

\section{Acknowledgments}

This work was carried out with the guidance of Barak Kol. I am also thankful to my longtime collaborator Shahar Hadar.

\section{References}

1. O. Birnholtz, Gravitational Waves: Sources and Calculational Methods - From Astrophysics to Field Theory and Back, Ph.D thesis, Hebrew University of Jerusalem, Israel, 2015.

2. O. Birnholtz, S. Hadar and B. Kol, Phys. Rev. D 88, 104037 (2013) [arXiv:1305.6930 [hep-th]].

3. O. Birnholtz, S. Hadar and B. Kol, Int. J. Mod. Phys. A 29, no. 24, 1450132 (2014) [arXiv:1402.2610 [hep-th]].

4. O. Birnholtz and S. Hadar, Phys. Rev. D 89, no. 4, 045003 (2014) [arXiv:1311.3196 [hep-th]].

5. O. Birnholtz and S. Hadar, Phys. Rev. D 91, no. 12, 124065 (2015) doi:10.1103/PhysRevD.91.124065 [arXiv:1501.06524 [gr-qc]].

6. O. Birnholtz, Int. J. Mod. Phys. A 30, no. 02, 1550011 (2015)doi:10.1142/ S0217751X15500116 [arXiv:1410.5871 [physics.class-ph]].

7. L. Blanchet, T. Damour, G. Esposito-Farese and B. R. Iyer, Phys. Rev. D 71, 124004 (2005) [gr-qc/0503044].

8. B. Kol and M. Smolkin, Class. Quant. Grav. 25, 145011 (2008) [arXiv:0712.4116 [hep-th]].

9. J. S. Schwinger, J. Math. Phys. 2, 407 (1961).

10. C. R. Galley, Phys. Rev. Lett. 110, no. 17, 174301 (2013) [arXiv:1210.2745 [gr-qc]].

11. V. Cardoso, O. J. C. Dias and J. P. S. Lemos, Phys. Rev. D 67, 064026 (2003) [hepth/0212168].

12. V. Cardoso, O. J. C. Dias and P. Figueras, Phys. Rev. D 78, 105010 (2008) [arXiv:0807.2261 [hep-th]]. 